\title{
Drug delivery systems for ovarian cancer treatment: a systematic review and meta-analysis of animal studies
}

René Raavé, Rob BM de Vries, Leon F Massuger, Toin H van Kuppevelt, Willeke F Daamen

Current ovarian cancer treatment involves chemotherapy that has serious limitations, such as rapid clearance, unfavorable biodistribution and severe side effects. To overcome these limitations, drug delivery systems (DDS) have been developed to encapsulate chemotherapeutics for delivery to tumor cells. However, no systematic assessment of the efficacy of chemotherapy by DDS compared to free chemotherapy (not in a DDS) has been performed for animal studies. Here, we assess the efficacy of chemotherapy in DDS on survival and tumor growth inhibition in animal studies. We searched PubMed and EMBASE (via OvidSP) to systematically identify studies evaluating chemotherapeutics encapsulated in DDS for ovarian cancer treatment in animal studies. Studies were assessed for quality and risk of bias. Study characteristics were collected and outcome data (survival/hazard ratio or tumor growth inhibition) were extracted and used for meta-analyses. Meta-analysis was performed to identify and explore which characteristics of DDS influenced treatment efficacy. A total of 44 studies were included after thorough literature screening (2735 studies found after initial search). The risk of bias was difficult to assess, mainly because of incomplete reporting. A total of 17 studies ( 377 animals) and 16 studies (259 animals) could be included in the meta-analysis for survival and tumor growth inhibition, respectively. In the majority of the included studies chemotherapeutics entrapped in a DDS significantly improved efficacy over free chemotherapeutics regarding both survival and tumor growth inhibition. Subgroup analyses, however, revealed that cisplatin entrapped in a DDS did not result in additional tumor growth inhibition compared to free cisplatin, although it did result in improved survival. Micelles did not show a significant tumor growth inhibition compared to free chemotherapeutics, which indicates that micelles may not be a suitable DDS for ovarian cancer treatment. Other subgroup analyses, such as targeted versus non-targeted DDS or IV versus IP administration route, did not identify specific characteristics of DDS that affected treatment efficacy. This systematic review shows the potential, but also the limitations of chemotherapy by drug delivery systems for ovarian cancer treatment. For future animal research, we emphasize that data need to be reported with ample attention to detailed reporting. 


\section{Drug delivery systems for ovarian cancer treatment: a systematic review and meta-analysis of animal studies}

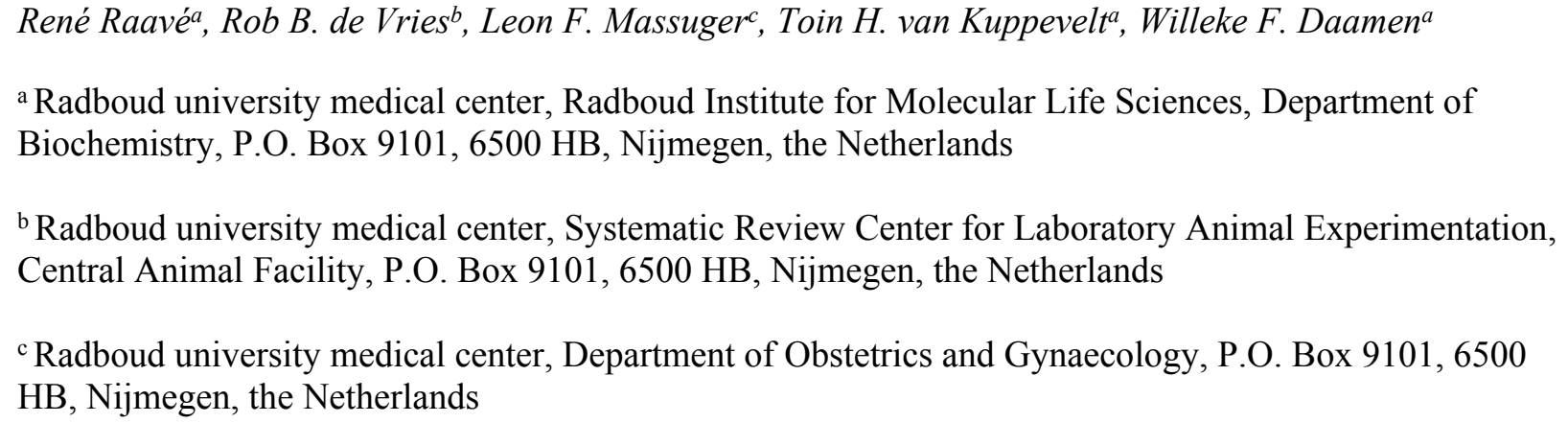

\section{Corresponding author}

Willeke F. Daamen PhD

Willeke.Daamen@Radboudumc.nl

Telephone: +31243614303

Fax: +31 243616413

Radboud university medical center

Radboud Institute for Molecular Life Sciences

Department of Biochemistry (280)

P.O. Box 9101

6500 HB Nijmegen

The Netherlands

\section{Email addresses authors}

René Raavé, Rene.Raave@radboudumc.nl

Rob B. de Vries, Rob.deVries@radboudumc.nl

Leon F. Massuger, Leon.Massuger@radboudumc.nl

Toin H. van Kuppevelt, Toin.vanKuppevelt@radboudumc.nl

Willeke F. Daamen, Willeke.Daamen@radboudumc.nl 


\section{$37 \quad 1 \quad$ Abstract}

38 Current ovarian cancer treatment involves chemotherapy that has serious limitations, such as rapid

39 clearance, unfavorable biodistribution and severe side effects. To overcome these limitations, drug

40 delivery systems (DDS) have been developed to encapsulate chemotherapeutics for delivery to tumor

41 cells. However, no systematic assessment of the efficacy of chemotherapy by DDS compared to free

42 chemotherapy (not in a DDS) has been performed for animal studies. Here, we assess the efficacy of

43 chemotherapy in DDS on survival and tumor growth inhibition in animal studies.

44 We searched PubMed and EMBASE (via OvidSP) to systematically identify studies evaluating

45 chemotherapeutics encapsulated in DDS for ovarian cancer treatment in animal studies. Studies were

46 assessed for quality and risk of bias. Study characteristics were collected and outcome data

47 (survival/hazard ratio or tumor growth inhibition) were extracted and used for meta-analyses. Meta-

48 analysis was performed to identify and explore which characteristics of DDS influenced treatment

49 efficacy.

50 A total of 44 studies were included after thorough literature screening (2735 studies found after

51 initial search). The risk of bias was difficult to assess, mainly because of incomplete reporting. A total of

5217 studies (377 animals) and 16 studies (259 animals) could be included in the meta-analysis for survival

53 and tumor growth inhibition, respectively. In the majority of the included studies chemotherapeutics

54 entrapped in a DDS significantly improved efficacy over free chemotherapeutics regarding both survival

55 and tumor growth inhibition. Subgroup analyses, however, revealed that cisplatin entrapped in a DDS did

56 not result in additional tumor growth inhibition compared to free cisplatin, although it did result in

57 improved survival. Micelles did not show a significant tumor growth inhibition compared to free

58 chemotherapeutics, which indicates that micelles may not be a suitable DDS for ovarian cancer treatment.

59 Other subgroup analyses, such as targeted versus non-targeted DDS or IV versus IP administration route,

60 did not identify specific characteristics of DDS that affected treatment efficacy. 
61

This systematic review shows the potential, but also the limitations of chemotherapy by drug

62 delivery systems for ovarian cancer treatment. For future animal research, we emphasize that data need to

63 be reported with ample attention to detailed reporting.

64 Keywords: animal studies, drug delivery systems, meta-analysis, ovarian cancer, systematic review 65 


\section{Introduction}

Ovarian cancer is the most lethal of all gynecological cancers. It is estimated that approximately 65,500 women were diagnosed with ovarian cancer and that about 42,700 women deceased due to ovarian cancer in Europe in 2012 (Ferlay et al. 2013). Conventional therapy includes neoadjuvant chemotherapy with subsequent surgical interval debulking and subsequent chemotherapy or primary surgical debulking with adjuvant chemotherapy (Vergote et al. 2011; Vergote et al. 2010). Although systemic intravenous administration of chemotherapeutics results in elimination of cancer cells, it is associated with serious shortcomings. Chemotherapeutics have a short half-life, are toxic to healthy cells and show an unfavorable biodistribution resulting in undesired side effects such as bone-marrow suppression, neuropathy, cardiotoxiticy, hair loss and nausea (Bergkvist \& Wengstrom 2006; Chon et al. 2012; Love et al. 1989; Massey et al. 2014; Monsuez et al. 2010; Truong et al. 2014). Moreover, next to systemic intravenous (IV) administered chemotherapy, local intraperitoneal (IP) in combination with IV administration is applied as well and was found to increase survival time in ovarian cancer patients (Armstrong et al. 2006; Barlin et al. 2012; Jaaback et al. 2011), but these patients had more side effects. Drug delivery systems (DDS) may overcome the current disadvantages of chemotherapeutics. By encapsulating toxic chemotherapeutics, DDS are designed to increase concentrations of chemotherapeutics at the tumor site, which could eventually result in higher treatment efficacy, while simultaneously reducing exposure of chemotherapeutics to healthy cells, resulting in a therapy with reduced side effects.

To date, abundant research has been performed on DDS, which has resulted in many kinds of DDS, such as liposomes, micelles or 'nanoparticles' (Lammers et al. 2012; Tomasina et al. 2013), with different characteristics for treatment of various types of cancer, including ovarian cancer. Several factors may affect the efficacy of DDS. For instance, size can be of importance as for long blood-circulation times and optimal tumor penetration an optimal size range of DDS is estimated to be in the sub-100 nm, but not smaller than $6 \mathrm{~nm}$ to prevent unwanted removal (Perrault et al. 2009). Another parameter that is often 
92 varied among DDS is PEGylation, which is intended to prevent unwanted uptake by the liver and spleen

93 by coating the surface of DDS with poly(ethylene)glycol (PEG) resulting in increased blood-circulation

94 times (Ernsting et al. 2013; Perrault et al. 2009). With increasing circulation time, increased accumulation

95 of DDS can be found at the tumor site. By the enhanced permeability and retention (EPR) effect of the

96 tumor cell aggregates, due to leaky blood vessels, DDS accumulate in the tumor area and release their

97 content, so-called passive targeting (Iyer et al. 2006). On the other hand, a more active way of targeting

98 can be achieved by conjugating anti-tumor antibodies or specific receptor ligands to the wall of capsules

99 to target tumor cells specifically (Danhier et al. 2010). The passive and active targeting strategies mainly

100 apply to intravenously (IV) administered DDS. However, as IP administered chemotherapy in

101 combination with IV administered chemotherapy is being clinically applied, DDS are also being

102 administered IP instead of IV in ovarian cancer (Gunji et al. 2013; Ye et al. 2013; Zhang et al. 2013),

103 introducing another variable in DDS that can affect the efficacy of DDS therapies. Furthermore, the DDS

104 preparation material can be varied from metals to polymers to proteins, which influences properties such

105 as biodegradability, immunogenicity and toxicity, but also drug release characteristics or cellular uptake

106 of DDS. Various chemotherapeutics are entrapped in DDS for ovarian cancer treatment, such as cisplatin,

107 paclitaxel or doxorubicin, affecting the outcome of DDS treatment as well. All in all, preclinical studies

108 showed that many parameters can be varied in DDS. It is still unclear, however, which variant is most

109 effective.

110

111 The majority of DDS are evaluated in vitro before being tested in animal models using different cancer

112 cell lines. In vivo evaluation has shown a wide range of therapeutic efficacies, with different treatment

113 regimes and several time periods. Several reviews describe possible improved efficacies that

114 chemotherapy by DDS may have in animal models for cancers such as breast cancer (Yezhelyev et al.

115 2006), lung cancer (Loira-Pastoriza et al. 2014), melanoma (Cheng et al. 2014), brain cancer (Chen et al.

116 2013), colorectal cancer (De Smet et al. 2013) and ovarian cancer (Tomasina et al. 2013). A recent

117 literature overview by Tomasina et al. showed a number of DDS that have been studied for ovarian 
118 cancer treatment (Tomasina et al. 2013). However, no systematic assessment of the efficacy of DDS in 119 experimental ovarian cancer, or other cancer types, and the effects of the different characteristics of these

120 DDS on treatment outcome has been reported. Therefore, we have conducted a systematic review of 121 animal studies in order to gain insight into the effectiveness of the many types of DDS tested for ovarian 122 cancer treatment.

124 In clinical studies, systematic reviews are common practice and they are also gaining popularity in preclinical (animal) studies. Compared to narrative reviews, systematic reviews are more structured and more thorough, resulting in a more comprehensive and transparent overview. Systematic reviews are therefore an ideal method for gaining a better understanding of the role DDS play in ovarian cancer therapy. Furthermore, such review may give new insights into the most effective capsule characteristics, how to improve the use and design of animal models, and eventually clinical trials. Moreover, metaanalysis can be used as an additional tool in systematic reviews of animal studies. While in meta-analyses of clinical data the primary goal is mostly to obtain a precise estimate of the overall effect of a certain intervention, in meta-analyses of animal studies the exact overall effect size may not be that informative (because of the often large heterogeneity between animal studies) and therefore the goal is of explorative nature to identify factors that affect the main outcome (Hooijmans et al. 2014a).

In this article, we report the results of the first systematic review of DDS evaluated in ovarian cancer animal models. In a comprehensive literature screening, we included all animal studies that used chemotherapeutics encapsulated in a DDS and evaluated their therapeutic efficiency in an orthotopic ovarian cancer animal model. A complete overview of the available literature including an assessment of the risk of bias of the individual studies is included. Where possible, meta-analyses were performed to study the extent to the efficacy of DDS depend on the different subgroup characteristics (type of drug delivery system, targeted vs. non-targeted DDS, IP vs. IV administration, type of xenografted cell line and type of chemotherapeutic in DDS). 


\section{Methods}

\section{$146 \quad 3.1 \quad$ Search strategy, inclusion and exclusion criteria}

147 To include as many animal studies as possible on drug delivery systems for ovarian cancer treatment, a 148 comprehensive search strategy for PubMed and EMBASE (via OvidSP) was developed. The search 149 strategy consisted of three specific search components addressing: 1) drug delivery systems; 2) ovarian 150 cancer; and 3) animal studies. The search strategy included thesaurus terms and keywords on the subject 151 of drug delivery systems and ovarian cancer (see supplemental methods for complete search strings). To 152 include all animal studies, previously developed PubMed and EMBASE search filters were used (de Vries 153 et al. 2014; Hooijmans et al. 2010). No language restrictions were applied.

After the search strategy had been executed in PubMed and EMBASE (search up until September $1^{\text {th }}$ 2014), duplicates were manually removed and the resulting studies were screened by title and abstract and classified as included, more information required or excluded, according to predefined exclusion criteria to exclude studies that did not comply with our research question (see supplemental methods for criteria). Included and more information required classified studies were subjected to a full text screening using additional exclusion criteria described in the supplemental methods. Screenings were performed independently by two reviewers (RR and WD) using Early Systematic Review Software 2.0 (EROS, Institute of Clinical Effectiveness and Health Policy, Buenos Aires, Argentina). Differences in classification between reviewers were discussed until consensus was reached. Studies in a language other than English (e.g. Japanese and Chinese) were screened by title and abstract by native speakers for that specific language. If a non-English study was included in the systematic review, it was professionally translated by "Radboud in'to Languages" (Radboud University, the Netherlands). 


\section{$168 \quad 3.2 \quad$ Study characteristics}

169 Journal and author information from all included studies was registered. Drug delivery characteristics 170 (e.g. material, size, etc.), animal model information (e.g. species, cell lines, etc.) and treatment and 171 outcome characteristics (e.g. dose, regime, tumor size evaluation, etc.) were extracted. Conference 172 abstracts and studies without data comparing free drug vs. encapsulated drug were not included in the 173 meta-analyses. One study (Ueno 1988) was not included in the meta-analysis as we were not able to 174 identify the specific inoculation area (subcutaneous or intraperitoneal).

\subsection{Risk of bias analysis}

177 To gain insight into the methodological quality of the included studies, we performed a risk of bias 178 assessment according to an adapted version of the risk of bias tool developed by Hooijmans et al. 2014 179 (Hooijmans et al. 2014b). Questions regarding reporting of randomization, blinding and sample size calculation were added to the items from the risk of bias tool (see supplemental methods for complete list). The complete list included 12 questions about the study quality such as "Was the allocation adequately concealed?' and “Were incomplete outcome data adequately addressed?". Since we were only between the reviewers were discussed until consensus was reached.

\subsection{Data extraction and statistical analyses}

188 For statistical analysis, two outcome measures that were presented frequently among the included studies were selected; survival (time-to-event data) and tumor growth inhibition. 
191 Studies presenting survival data included experiments that show differences in survival of animals during

192 the course of the study between the treatment conditions; chemotherapeutics administered in a DDS and

193 chemotherapeutics administered without a DDS (free drug control). Tumor inhibition data were expressed

194 in the studies as decrease in tumor size measured by, for instance, tumor weight or bioluminescence

195 signal from the inoculated ovarian cancer cells.

196

197 To compare each study's result, data was extracted from the included studies. From experiments with

198 survival data, individual time-to-event data was extracted and from experiments with tumor growth

199 inhibition data we extracted the raw data such as tumor weight or bioluminescence signal. If these data

200 were only depicted graphically, authors were contacted by e-mail to provide the numerical data. If the

201 requested data could not be provided, we extracted individual time-to-event survival data or tumor growth

202 inhibition means with SD and the number of animals using ImageJ (1.46r, National Institutes of Health,

203 USA).

204

205 Since raw time-to-event survival data by themselves cannot be used for meta-analysis, hazard ratios were 206 calculated. Hazard ratios represent the risk of dying over the course of the experiment. A hazard ratio $>1$ 207 indicates that animals have a higher chance of dying due to their experimental condition, while a hazard 208 ratio $<1$ indicates that animals have less chance of dying over the course of the experiment due to their treatment condition. If numerical hazard ratios were presented in included studies, they were used directly without further processing for meta-analysis. All graphically extracted survival data were first analyzed using SPSS Statistics 20.0.01 software (IBM, Amsterdam, the Netherlands). Log-hazard ratios and

212 standard errors were determined using a Cox regression analysis with treatment conditions set as 213 categorical covariates. Free drug control conditions (chemotherapeutic not in a DDS) were set as 214 reference category. To compare results between studies with tumor growth outcome measures, data were

215 translated into standardized mean differences (SMD; experimental group mean minus control group mean

216 divided by the pooled standard deviations of the two groups). A negative SMD indicates a larger 
217 inhibition of tumor growth due to treatment with DDS compared to free drugs (not in a DDS), while a 218 positive SMD value indicates that treatment with free drugs is more effective. Means, standard deviations

219 (SDs) and the number of animals were extracted from the experiments and used to calculate SMDs.

220

221

222

223

224

225

226

227

228

Meta-analyses were performed using Review Manager Version 5.1 (Copenhagen, The Nordic Cochrane Centre, The Cochrane Collaboration, 2011). Two separate meta-analyses were performed for the outcome measures survival and tumor growth inhibition. For time-to-event data (survival), a (generic) inverse variance model with random effects and hazard ratio as effect measure was applied. In this model, the extracted log-hazard ratios with standard errors from the studies were entered in Review Manager and used to calculate hazard ratios with $95 \%$ confidence intervals for the meta-analysis. For tumor growth inhibition data, a (continuous) inverse variance model with random effects and standardized mean difference as effect measure was used. If the same study included more than two experimental conditions, the separate experiments were included in the meta-analysis. If in these cases there was only one control condition, the $n$ for the control condition was adjusted by dividing it by the number of included conditions, to prevent that animals were included more than once in the meta-analysis. $\mathrm{I}^{2}$ was used as a measure of heterogeneity. In order to explore potential causes of heterogeneity, subgroup analyses were planned for 1) drug delivery system, 2) chemotherapeutic used, 3) xenografted cell line in animal model, 4) targeted vs. non-targeted and 5) IP vs. IV administered DDS. Because of a lack of power, subgroups containing less than three experiments were not used for subgroup analysis. To further investigate the effect of individual experiments on the overall effect or on subgroup effects, sensitivity analyses were performed by checking whether the direction of the overall or subgroup effect and their confidence intervals altered substantially when individual experiments were removed from the meta-analyses.

Furthermore, to identify possible publication bias (an underrepresentation of small studies with neutral or negative effects), a funnel scatter plot with the studies' intervention effect on the horizontal axis and the studies' standard error on the vertical axis was created and evaluated. 


\section{$244 \quad 4$ Results}

\section{$245 \quad 4.1 \quad$ Study inclusion and characteristics}

246 Search strategies designed to include animal studies about ovarian cancer and treatment using drug 247 delivery systems resulted in a total of 2735 studies, whereof 1682 and 1053 from EMBASE and PubMed, 248 respectively (Fig. 1). After removal of duplicates, 1947 studies were screened by title and abstract, which 249 resulted in removal of 1682 studies. Subsequently, 265 studies were screened by full text. Of the studies screened by full text, 221 studies were excluded and 44 were included in this systematic review. The major reason for excluding studies was the use of a clinically irrelevant animal model ("ovarian cancer cells used in other area than peritoneal cavity or ovaries").

The characteristics of the included studies are summarized in supplementary table 1. Many different DDS were designed and used to treat ovarian cancer in vivo. Most studies (36\%) used liposomes to encapsulate a chemotherapeutic drug. Approximately $16 \%$ of the studies used micelles while others used capsules labeled as nano- or microparticles (9\% and 27\%, respectively). Furthermore, studies that used nanogels, nanosuspensions, microbullets, virus cages and nanobins were included as well. Preparation material varied among the different designed DDS as shown in supplementary table 1. Active targeting to ovarian cancer cells using antibodies and receptor ligands such as HER-2 (Cirstoiu-Hapca et al. 2010), OV-TL3 (Storm et al. 1994; Vingerhoeds et al. 1996), folate (Chaudhury et al. 2012; Tong et al. 2014; Werner et al. 2011; Zeng et al. 2013) or luteinizing hormone-releasing hormone analogs (Pu et al. 2014) conjugated to the DDS were used in $30 \%$ of the included studies.

Several studies applied specific modifications to create a triggered drug-release. Gilmore et al. prepared nanoparticles from an acrylate monomer to create particles that are stable at neutral $\mathrm{pH}$ and expand after 
269

270

271

272

273

274

275

276

277

278

279

280

281

282

283

284

285

286

287

288

289

290

291

292

293

294

that also released its payload at low $\mathrm{pH}$ (Xu et al. 2006). Moreover, using a poly-isobutylene-maleicglucosamine cisplatin combination, an acid-triggered drug delivery system was developed and probed to treat ovarian cancer by Paraskar et al. and Sengupta et al. (Paraskar et al. 2010; Sengupta et al. 2012).

Other modifications were applied to ensure specific delivery and release of anti-tumor drug to ovarian cancer cells and thus to increase the efficiency of the DDS in vivo. Lu et al. designed two types of tumor penetrating microparticles from poly(DL-lactide-coglycolide) that could either prime tumors with a rapid release, or sustain a specific drug level using a slow release microparticle (Lu et al. 2008). Others applied a post-ultrasound strategy to release the chemotherapeutic drug from micelles or to facilitate intracellular drug uptake from microbubbles upon injection (Gao et al. 2005; Pu et al. 2014; Rapoport et al. 2004).

Frequently used clinically approved chemotherapeutic agents for ovarian cancer treatment doxorubicin, cisplatin and paclitaxel were used in $27 \%, 16 \%$ and $36 \%$ of the studies, respectively. The remainder used other chemotherapeutic agents as described in supplementary table 1. One study applied co-delivery of doxorubicin and irinotecan using liposomes (Javid et al. 2014).

Other smaller parameters were applied to the DDS as well. About $32 \%$ of the included studies applied PEGylation to prolong circulation time. The route of application was varied among the included studies. DDS were either administered intraperitoneally (68\%), intravenously (18\%), or a combination of both $(14 \%)$.

About $7 \%$ of the studies used a rat (Fisher F344, female) model in combination with the NuTu19 rat ovarian cancer cell line, while the remaining $(93 \%)$ used a mouse model that was either $(73 \%)$, male $(2 \%)$, a combination of male and female male $(2 \%)$ or not described $(23 \%)$. Within the mice studies, the strains and genotypes varied a lot of which an athymic or nude (Foxn $\left.{ }^{n u}\right)$ mice lacking T-cells was most frequently used (64\%). Among the xenografted mice models, most were inoculated with well-established 
295

296

297

298

299

300

301

302

303

304

305

306

307

308

309

310

311

312

\section{$313 \quad 4.3 .1 \quad$ Survival}

\section{4.3.1.1 Forest plots}

31518 studies described results with survival data. These data were used to calculate hazard ratios. A total of

31630 experiments were suitable for performing a meta-analysis, which represented 377 animals. From these

31730 experiments, 12 experiments showed a significantly decrease in hazard ratio, while one experiment 
318 showed a significant increase in hazard ratio (Fig. 3a). This may indicate that treatment of animal models

319 for ovarian cancer with chemotherapeutics in a DDS is more effective in preventing death than treatment

320 with free chemotherapeutics. For four studies (due to small group numbers) no models could be fitted,

321 which resulted in a hazard ratio of 0 with a very wide confidence interval.

\section{4.3.1.2 Type of DDS}

323 As shown in Figure 3b, a subgroup analysis was performed to evaluate the overall effect of experiments

324 that used liposomes (12 experiments) or micro/nanocapsules (15 experiments). No difference in effect on

325 hazard ratio was found between experiments that used liposomes or micro/nanocapsules; all resulted in a

326 significant decrease of the hazard ratio.

\section{4.3.1.3 Type of chemotherapeutic}

328 To investigate whether different tumor drugs encapsulated in DDS affect the hazard ratio, subgroup 329 analysis by chemotherapeutic cisplatin (7 experiments), doxorubicin (4 experiments) and paclitaxel (16

330 experiments) was performed (Fig. 3b). Cisplatin, doxorubicin and paclitaxel all resulted in a significant

331 decrease in hazard ratio. No significant differences were observed among the three drug subgroups.

332

\subsubsection{Targeting vs. non-targeting}

Drug delivery systems targeted specifically (12 experiments) to ovarian cancer cells did not result in a lower hazard ratio compared to non-targeted DDS (18 experiments). Both treatment strategies resulted in a lower subtotal hazard ratio, suggesting that both targeted and non-targeted DDS treatment result in improved survival rates (Fig. 3b).

\subsubsection{Route of administration}

A subgroup analysis of the different routes of administration was performed to explore whether this would affect the treatment outcome. Both IP (17 experiments) and IV (7 experiments) administration significantly lowered the risk of dying over time (Fig. 3b). Moreover, experiments that used a 
342 combination strategy of IP and IV treatment (6 experiments) also resulted in a lower hazard ratio. No

343 statistical differences between IV, IP or a combination of IV and IP administration were observed.

\subsubsection{Applied xenografted cell line}

346 Ovarian cancer cell lines SKOV-3 (9 experiments), OVCAR-3 (5 experiments), A2780 (7 experiments), ID-8 (3 experiments) and IGROV-1 (3 experiments) subgroups could be included in the subgroup analysis as these had $\geq 3$ studies in the several subgroups. This meta-analysis showed that mice xenografted with SKOV-3, OVCAR-3 and ID-8 followed by treatment with chemotherapeutics had a significant decrease in hazard ratio (Fig. 3b). Mice xenografted with IGROV-1 or A2780 that were treated with DDS did not significantly benefit from DDS treatment compared to free drug controls.

\subsubsection{Tumor growth inhibition}

354 inhibition than free chemotherapeutics.

\subsubsection{Forest plot}

A total of 16 studies presented data regarding tumor growth inhibition using a drug delivery system compared to a free drug control. From these studies, 21 experiments could be used for meta-analysis representing a total of 259 animals. Nine of the experiments showed a statistically significant result to the effect that chemotherapeutics in DDS inhibit tumor growth better than free drugs (Fig. 4a). The study of Konishi et al. reported a significant tumor growth inhibition. However, this could not be included in the meta-analysis due to the absence of a standard deviation in the experimental group. No studies reported significantly more tumor growth inhibition by free drug treatment compared to the DDS treatment. These results suggest that chemotherapeutics in a DDS in general have a higher efficacy regarding tumor growth 
365

366

367

368

369

370

371

372

373

374

375

376

377

378

379

380

381

382

383

384

385

\subsubsection{Type of DDS}

To gain insight in the effectiveness of different types of DDS, a subgroup analysis by DDS type was performed (Fig 4b). A statistically significant difference between the subgroups micro/nano-particles (13 experiments) and micelles (3 experiments) was observed; treatment with micro/nano-particles seemed to perform better than treatment with micelles. On the other hand, no significant difference between the results of liposomes (3 experiments) and micro/nanoparticles was found.

\subsubsection{Type of chemotherapeutic}

Subgroup analysis of tumor growth inhibition data by anti-tumor drug was possible for the chemotherapeutics cisplatin ( 7 experiments) and paclitaxel (9 experiments) with 7 and 9 experiments, respectively (Fig. 4b). Surprisingly, cisplatin encapsulated in DDS did not result in enhanced tumor growth inhibition compared to free drug control, whereas encapsulated paclitaxel was much more effective than free paclitaxel. Moreover, the difference between subgroups paclitaxel and cisplatin was statistically significant.

\subsubsection{Targeted vs. non-targeted}

Non-targeted DDS reach tumor cells passively by exploiting the leaky vessels of the tumor vasculature.

On the other hand, DDS can be decorated with tumor-specific antibodies or receptor-ligands to actively target tumor cells. A subgroup analysis for targeted (4 experiments) vs. non-targeted (17 experiments) DDS showed that both targeted and non-targeted DDS could significantly inhibit tumor growth more compared to their free drug controls (Fig 4b). However, no significant difference was observed between the targeted and non-targeted subgroups. 
386

387

388

389

390

391

392

393

394

395

396

397

398

399

400

401

402

403

404

405

406

407

\subsubsection{Route of administration}

A total of 16 experiments administered their treatment IP, while 4 experiments used an IV strategy. Both routes seem to be effective, but no statistical difference in effectiveness between the two routes was found, suggesting that IP administration of DDS has no advantage over IV in animals.

\subsection{Sensitivity analysis}

To assess the robustness of the meta-analyses' results, a sensitivity analysis was performed. This analysis assessed the influence of individual studies with their specific experimental set-up (e.g. number and type of inoculated ovarian cancer cells, treatment dose and regime, or genotype differences) on the overall outcome effect.

\subsubsection{Survival data}

It was investigated whether studies that had dose differences between the DDS and free drug groups (marked with an asterisk in Figure 3 and 4) affected the overall effect. Exclusion of these studies, however, did not affect the direction of the overall effect.

(1)

For experiments from Chaudhury et al. (one experiment), Cirstoiu-Hapca et al. (two experiments), and Yang et al. (one experiment), it was not possible to accurately estimate a hazard ratio from the log-hazard ratios. In these experiments, there was not enough information (e.g. only one event over the course of the experiment) to converge and fit a model. This resulted in a hazard ratio of 0 with a very wide confidence interval. Excluding these experiments from the analysis hardly had any effect on the overall outcome. 


\subsubsection{Tumor growth inhibition data}

409 For tumor growth inhibition data, experiments from Javid et al. and Lu et al. 2007 showed extremely high 410 tumor growth inhibition for their DDS groups. Therefore, we wondered whether the overall positive effect 411 was caused by these experiments. However, these studies did only affect overall tumor growth inhibition 412 to a small extent; a meta-analysis without these studies still resulted in a significant inhibition of tumor 413 growth due to treatment with chemotherapeutics entrapped in a DDS.

414

415 Li and Howell, and Patankar et al. used different doses of chemotherapeutics in the treatment group and control group. Therefore, it was tested whether these studies were responsible for the positive overall outcome. However, excluding these studies did not affect the overall meta-analysis effect size.

Moreover, it was investigated whether two studies that used a rat model instead of a mouse model influenced the overall outcome (Ye et al. 2013 and Lu et al. 2007). A meta-analysis without these rat studies still resulted in an overall significant inhibition of tumor growth for animals treated with chemotherapeutics in a DDS compared to animals treated with free chemotherapeutics.

\subsection{Publication bias assessment}

Publication bias was assessed for the time-to-event outcome measure, since this outcome measure included the largest number of studies. To investigate publication bias, a funnel plot was created (Fig. 5). The experiments with almost infinite confidence intervals (Chaudhury et al., Cirstoiu-Hapca et al. and Yang et al.,) were not included in the funnel plot as these would introduce a very large y-axis interval making the graph unclear. The funnel plot indicated missing studies at the right bottom side of the overall

430 effect where small studies with a high hazard ratio (less survival in DDS group) would be expected, 431 suggesting publication bias. 


\section{Discussion}

433 This systematic review was performed to investigate the effect of chemotherapeutic-DDS and their 434 specific characteristics on ovarian cancer treatment in animal models. We looked at two outcome 435 measures; survival and tumor growth inhibition, which resulted in meta-analyses of 17 and 16 studies that 436 included 377 and 259 animals, respectively. Overall, the majority of the studies showed that treatment 437 with chemotherapeutics entrapped in DDS used for in vivo treatment of experimental ovarian cancer had 438 better efficacies on both survival and tumor growth inhibition compared to chemotherapeutics not 439 entrapped in a DDS. This result is to some extent similar to what is found in clinical studies, which 440 observed increased efficacy of doxorubicin in a DDS (pegylated liposomes) either in different staged 441 ovarian cancer patient groups or compared to different treatment regimes with other chemotherapeutics. 442 Although these studies did not compare free doxorubicin and doxorubicin by a DDS, most consider 443 pegylated liposomal doxorubicin as a safe and effective treatment (Gordon et al. 2000; Muggia et al. 444 1997; Safra et al. 2001; Uziely et al. 1995).

However, a few observations in the field of drug delivery and ovarian cancer treatment were not supported by our results. Our results in animal studies do not show that one administration route (either IV, IP or a combination of both) had an advantage over another route looking at tumor growth inhibition and survival. This seems to be in contrast with clinical data where several lines of evidence suggest that treatment of ovarian cancer patients with a combination of IP and IV treatment with free chemotherapeutics may be more effective than IV treatment only (Jaaback et al. 2011). It should be taken into account that these clinical studies were not performed with DDS and always included an additional systemic chemotherapy over the IP therapy. This may explain the lack of improved efficacy by IP treatment over IV treatment in our meta-analysis.

An interesting observation is that our results suggest that cisplatin, a first choice chemotherapeutic for ovarian cancer treatment, may not be a suitable candidate for treatment of ovarian cancer using DDS, 
458 since cisplatin in DDS did not lead to more tumor growth inhibition than free cisplatin. However, this was

459 not the case for survival, a clinically more important outcome measure, where all chemotherapeutics in

460 DDS resulted in a significant improvement of survival compared to free chemotherapeutics. It should be

461 noted that results from tumor growth inhibition and survival outcome measures were mostly not based on

462 data from the same studies. Interestingly is that in a phase II clinical study evaluating liposomal cisplatin

463 a lack of clinical response was observed (Seetharamu et al. 2010). Moreover, in 1998, Sugiyama et al.

464 evaluated microspheres containing cisplatin compared to an aqueous solution of cisplatin and found in a

465 small ovarian cancer patient group similar toxicity profiles, but no data on efficacy was shown (Sugiyama

466 et al. 1998). No subsequent phase I/II clinical trials of this DDS regarding ovarian cancer treatment could

467 be identified in current literature, which may suggest a possible lack of clinical outcome. These two

468 cisplatin DDS examples may confirm our results that cisplatin may not be the most suitable drug to be

469 used in a DDS for ovarian cancer treatment.

470

471 Our results show that animal studies do not indicate higher treatment efficacies by active targeting, as

472 both active and passive targeting resulted in almost similar inhibition of tumor growth and improved

473 survival in animal studies. This seems to be in contrast with the current direction of the drug delivery

474 research field where an important goal in the development of DDS is to improve treatment efficacy and

475 simultaneously decrease side effects of chemotherapeutics. By active targeting of tumor cells with

476 antibodies or tumor receptor ligands attached to DDS, it is hypothesized that these DDS only bind to

477 tumor cells and not to healthy cells, thereby improving treatment efficacy and simultaneously decreasing

478 side effects (Bae \& Park 2011). All 7 included studies in our systematic review that evaluated

479 chemotherapy by both targeted and non-targeted DDS did not show significant differences in survival or

480 tumor reduction meta-analyses. However, if targeted therapy would show an advantage over non-targeted

481 therapy, such as fewer side effects, chemotherapy by targeted DDS would be preferable over

482 chemotherapy by non-targeted DDS. Nevertheless, none of the included studies showed data on reduction

483 of side effects by targeted DDS. As our results showed no advantage of targeted DDS, although with 
484 limited power, we therefore carefully hypothesize that chemotherapy by targeted DDS may have no or 485 only little advantage over chemotherapy by non-targeted DDS when only looking at tumor growth 486 inhibition and survival. Future animal studies investigating differences between chemotherapy by targeted 487 and non-targeted DDS should be performed to show the advantages of targeted DDS.

Looking at tumor growth inhibition, our analysis suggested that micro/nanoparticle DDS are most efficient and significantly better than micelles. Micelles do not result in significant tumor growth inhibition, which suggests that micelles may not be the most suitable DDS for chemotherapeutic ovarian cancer treatment. This could not be confirmed with survival data, as the micelles subgroup contained too little experiments. The two experiments evaluating micelles and showing survival data both did not show a significant improved hazard ratio. Future research should therefore show whether chemotherapy using micelles would improve survival outcome. Moreover, we would like to emphasize that the micro/nanoparticle group was very heterogeneous. However, making subgroups of the micro/nanoparticle group was not feasible due to the lack of experiments performed with each specific DDS. Therefore, more DDS has the best efficacy.

We tried to investigate the role of the ovarian cancer animal model. During the screening of studies for inclusion in this systematic review, we came across many animal studies that used a less physiologically relevant subcutaneous animal model (Vanderhyden et al. 2003). As these animal models do not reflect the disease progression of ovarian cancer, we decided to focus only on studies that used a clinically important orthotopic intraperitoneal ovarian cancer animal model. This decision may explain why our results are less positive than the current direction in literature (e.g. no advantage of targeted DDS).

It is interesting that there is no consensus about the specific cell line used for the assessment of DDS 
510 (Domcke et al. 2013). Most frequently used ovarian cancer cells lines such as SKOV3, A2780 and

511 IGROV-1 may not be suitable models for ovarian carcinoma cell lines and results from experiments with

512 these cell lines should therefore be interpreted with caution, especially when translating these results to

513 the clinic.

514

515 Our results showed no significant improved survival in animal models with A2780 or IGROV-1 cell

516 lines. They may be considered to be poor models for ovarian cancer, but there are no explanations that

517 these cell lines would be less sensitive for chemotherapy by DDS. Despite to their lack of clinical

518 representativity, we have no reasons to prefer a certain cell type for experiments regarding chemotherapy

519 by DDS based on results from this systematic review and meta-analysis.

520

521 We want to mention a number of limitations of this review. Both the overall analysis and the subgroup

522

523

524

525

526

527

528

529

530

531

532

533

534

535 analyses displayed relatively high levels of heterogeneity, even though the levels within the subgroups were somewhat lower than in the overall analysis. Because of this (expected) heterogeneity, the metaanalyses were used to explore potential characteristics of DDS that affect final outcome in a hypothesisforming rather than hypothesis-confirming manner.

Another limitation is the lack of response from authors from included studies when asked to share their raw data. As only a few authors were willing to share their raw data, we had to extract raw data from most included studies manually. Although performed carefully, this may have introduced small errors in the data used for meta-analyses.

The possibility of bias in the included studies in this systematic review may have introduced an overestimation of the meta-analyses' results. The reliability of the results of a systematic review greatly depends on the quality of the included studies. Unfortunately, most studies lacked reporting of important details in their experimental set-up. Therefore, it was difficult to assess whether studies actually had a low 
536 or high risk of bias. To compare efficacies of chemotherapeutics in DDS compared to free

537 chemotherapeutics, the experimental set-up is of major importance. For instance, blinding and

538 randomization contribute to the overall validity of the experimental set-up (Hirst et al. 2014). Most

539 studies used humane endpoints for the sake of the animals' welfare. However, if not blinded, one can

540 imagine that control animals may be considered to meet humane endpoint criteria earlier (Bello et al.

541 2014), which may introduce a bias in the outcomes of the study, particularly if survival is an outcome

542 measure. Moreover, almost all studies used a xenografted animal model that was first inoculated with

543 cells before treatment initiation. Without any kind of randomization, differences in tumor baseline may be

544 introduced that could alter the final study outcome. In most of the included studies it was not mentioned

545 that blinding or randomization was performed, which may have introduced bias (Bebarta et al. 2003).

546 Moreover, to ensure enough power of an experimental design, power calculations are an essential tool.

547 None of the included studies described any kind of power calculation that may suggest lack of power in

548 the included studies. These possible overestimations by studies included with bias may implicate that our

549 observed effects may be less reliable. However, it may also be true that studies were correctly performed,

550 but that experiments were only poorly reported, which is known from previous systematic reviews on

551 animal studies that most studies poorly describe their in vivo experiments (Hooijmans et al. 2012).

552 Therefore we would like to encourage to improve reporting of animals studies by using for instance the

553 golden standard publication checklist (Hooijmans et al. 2011) or the ARRIVE guidelines (Kilkenny et al.

554 2010). Finally, a funnel scatter plot analysis suggests publication bias, which could have introduced an

555 overestimation of our results as well.

556

557 A major remark regarding our results is that we did not look at side effects as outcome measure. This

558 aspect may change the impact of our results. For instance, IP treatment in patients results in increased

559 survival, but these patients experience more severe side effects (e.g. pain, fatigue and gastrointestinal

560 effects (Armstrong et al. 2006; Barlin et al. 2012; Jaaback et al. 2011). If the application of

561 chemotherapeutics in DDS would decrease side effects in IP treatment, this may be a major improvement 
562 in patient quality of life. Moreover, results suggest that there is not a specific characteristic of DDS that 563 outperforms in tumor growth inhibition or survival. Again, if a specific characteristic of DDS would show 564 considerably less side effects, this class would be clinically very attractive although it does not 565 outperform other DDS regarding tumor size or survival in animal studies. The same is valid for the choice 566 of cytostatic drug. Our results do not suggest a specific higher efficacy for cisplatin, doxorubicin or 567 paclitaxel if entrapped in a DDS regarding survival in animal studies. However, if entrapment of one of 568 these drugs results in significant less side effects, this may be again of clinical importance and a major 569 argument to entrap this specific chemotherapeutic in a DDS, despite similar efficacies compared to the 570 other drugs as found in this systematic review. Although not in ovarian cancer, O'Brien showed that free 571 doxorubicin and pegylated doxorubicin in treatment of metastatic breast cancer showed comparable 572 overall survival with significantly less cardiotoxicity in the pegylated liposomal doxorubicin group 573 (O'Brien et al. 2004). As only a few studies included in this systematic review addressed side-effects, an additional new systematic review on animal studies with meta-analysis should be performed to assess the specific research question; the effect of entrapment of chemotherapeutics in DDS on side effects.

576

577 In conclusion, delivery of chemotherapeutics with a DDS seems to be effective with regard to both tumor 578 size and survival in animal models. Results of this study support the claim that delivery of 579 chemotherapeutics is more effective compared to treatment with free chemotherapeutics, and that this 580 efficacy is not dependent on specific characteristics of DDS. Future well-designed in vivo studies 581 evaluating the efficacy of different characteristics of DDS on tumor size inhibition, survival and side 582 effects should be performed to identify important characteristics of DDS for clinical translation. 


\section{$584 \quad 6 \quad$ Acknowledgements}

585 Gerrie Hermkens and Jos Peeters from the Radboudumc medical library are kindly acknowledged for 586 their help with identifying full text studies. Fang Yang and Shinju Takemoto (Dept. of Biomaterials, 587 Radboudumc) are both greatly acknowledged for their help with the screening of Chinese and Japanese 588 studies, respectively. Joanna in 't Hout (Dept. of Health Evidence) is acknowledged for her statistical 589 advice. 


\section{References}

Alagkiozidis I, Facciabene A, Carpenito C, Benencia F, Jonak Z, Adams S, Carroll RG, Gimotty PA, Hammond R, Danet-Desnoyers GA, June CH, Powell DJ, Jr., and Coukos G. 2009. Increased immunogenicity of surviving tumor cells enables cooperation between liposomal doxorubicin and IL-18. J Transl Med 7:104.

Armstrong DK, Bundy B, Wenzel L, Huang HQ, Baergen R, Lele S, Copeland LJ, Walker JL, Burger RA, and Gynecologic Oncology G. 2006. Intraperitoneal cisplatin and paclitaxel in ovarian cancer. N Engl J Med 354:34-43.

Bae YH, and Park K. 2011. Targeted drug delivery to tumors: myths, reality and possibility. $J$ Control Release 153:198-205.

Barlin JN, Dao F, Bou Zgheib N, Ferguson SE, Sabbatini PJ, Hensley ML, Bell-McGuinn KM, Konner J, Tew WP, Aghajanian C, and Chi DS. 2012. Progression-free and overall survival of a modified outpatient regimen of primary intravenous/intraperitoneal paclitaxel and intraperitoneal cisplatin in ovarian, fallopian tube, and primary peritoneal cancer. Gynecol Oncol 125:621-624.

Bebarta V, Luyten D, and Heard K. 2003. Emergency medicine animal research: does use of randomization and blinding affect the results? Acad Emerg Med 10:684-687.

Bello S, Krogsboll LT, Gruber J, Zhao ZJ, Fischer D, and Hrobjartsson A. 2014. Lack of blinding of outcome assessors in animal model experiments implies risk of observer bias. J Clin Epidemiol 67:973-983.

Bergkvist K, and Wengstrom Y. 2006. Symptom experiences during chemotherapy treatment--with focus on nausea and vomiting. Eur J Oncol Nurs 10:21-29.

Chaudhury A, Das S, Bunte RM, and Chiu GN. 2012. Potent therapeutic activity of folate receptortargeted liposomal carboplatin in the localized treatment of intraperitoneally grown human ovarian tumor xenograft. Int J Nanomedicine 7:739-751.

Chen J, Shao R, Zhang XD, and Chen C. 2013. Applications of nanotechnology for melanoma treatment, diagnosis, and theranostics. Int J Nanomedicine 8:2677-2688.

Cheng Y, Morshed RA, Auffinger B, Tobias AL, and Lesniak MS. 2014. Multifunctional nanoparticles for brain tumor imaging and therapy. Adv Drug Deliv Rev 66:42-57.

Chon SY, Champion RW, Geddes ER, and Rashid RM. 2012. Chemotherapy-induced alopecia. J Am Acad Dermatol 67:e37-47.

Cirstoiu-Hapca A, Buchegger F, Lange N, Bossy L, Gurny R, and Delie F. 2010. Benefit of anti-HER2coated paclitaxel-loaded immuno-nanoparticles in the treatment of disseminated ovarian cancer: Therapeutic efficacy and biodistribution in mice. J Control Release 144:324-331.

Danhier F, Feron O, and Preat V. 2010. To exploit the tumor microenvironment: Passive and active tumor targeting of nanocarriers for anti-cancer drug delivery. J Control Release 148:135-146.

De Smet L, Ceelen W, Remon JP, and Vervaet C. 2013. Optimization of drug delivery systems for intraperitoneal therapy to extend the residence time of the chemotherapeutic agent. ScientificWorldJournal 2013:720858.

de Vries RB, Hooijmans CR, Tillema A, Leenaars M, and Ritskes-Hoitinga M. 2014. Updated version of the Embase search filter for animal studies. Lab Anim 48:88.

Domcke S, Sinha R, Levine DA, Sander C, and Schultz N. 2013. Evaluating cell lines as tumour models by comparison of genomic profiles. Nat Commun 4:2126.

Ernsting MJ, Murakami M, Roy A, and Li SD. 2013. Factors controlling the pharmacokinetics, biodistribution and intratumoral penetration of nanoparticles. J Control Release 172:782-794.

Ferlay J, Steliarova-Foucher E, Lortet-Tieulent J, Rosso S, Coebergh JW, Comber H, Forman D, and Bray F. 2013. Cancer incidence and mortality patterns in Europe: estimates for 40 countries in 2012. Eur J Cancer 49:1374-1403.

Gao ZG, Fain HD, and Rapoport N. 2005. Controlled and targeted tumor chemotherapy by micellarencapsulated drug and ultrasound. J Control Release 102:203-222. 
639

640

641

642

643

644

645

646

647

648

649

650

651

652

653

654

655

656

657

658

659

660

661

662

663

664

665

666

667

668

669

670

671

672

673

674

675

676

677

678

679

680

681

682

683

684

685

686

687

688

Gilmore D, Schulz M, Liu R, Zubris KA, Padera RF, Catalano PJ, Grinstaff MW, and Colson YL. 2013. Cytoreductive surgery and intraoperative administration of paclitaxel-loaded expansile nanoparticles delay tumor recurrence in ovarian carcinoma. Ann Surg Oncol 20:1684-1693.

Gordon AN, Granai CO, Rose PG, Hainsworth J, Lopez A, Weissman C, Rosales R, and Sharpington T. 2000. Phase II study of liposomal doxorubicin in platinum- and paclitaxel-refractory epithelial ovarian cancer. J Clin Oncol 18:3093-3100.

Griset AP, Walpole J, Liu R, Gaffey A, Colson YL, and Grinstaff MW. 2009. Expansile nanoparticles: synthesis, characterization, and in vivo efficacy of an acid-responsive polymeric drug delivery system. J Am Chem Soc 131:2469-2471.

Gunji S, Obama K, Matsui M, Tabata Y, and Sakai Y. 2013. A novel drug delivery system of intraperitoneal chemotherapy for peritoneal carcinomatosis using gelatin microspheres incorporating cisplatin. Surgery 154:991-999.

Hirst JA, Howick J, Aronson JK, Roberts N, Perera R, Koshiaris C, and Heneghan C. 2014. The need for randomization in animal trials: an overview of systematic reviews. PLoS One 9:e98856.

Hooijmans C, de Vries R, Leenaars M, and Ritskes-Hoitinga M. 2011. The Gold Standard Publication Checklist (GSPC) for improved design, reporting and scientific quality of animal studies GSPC versus ARRIVE guidelines. Lab Anim 45:61.

Hooijmans CR, de Vries RB, Rovers MM, Gooszen HG, and Ritskes-Hoitinga M. 2012. The effects of probiotic supplementation on experimental acute pancreatitis: a systematic review and metaanalysis. PLoS One 7:e48811.

Hooijmans CR, IntHout J, Ritskes-Hoitinga M, and Rovers MM. 2014a. Meta-analyses of animal studies: an introduction of a valuable instrument to further improve healthcare. ILAR J 55:418-426.

Hooijmans CR, Rovers MM, de Vries RB, Leenaars M, Ritskes-Hoitinga M, and Langendam MW. 2014b. SYRCLE's risk of bias tool for animal studies. BMC Med Res Methodol 14:43.

Hooijmans CR, Tillema A, Leenaars M, and Ritskes-Hoitinga M. 2010. Enhancing search efficiency by means of a search filter for finding all studies on animal experimentation in PubMed. Lab Anim 44:170-175.

Iyer AK, Khaled G, Fang J, and Maeda H. 2006. Exploiting the enhanced permeability and retention effect for tumor targeting. Drug Discov Today 11:812-818.

Jaaback K, Johnson N, and Lawrie TA. 2011. Intraperitoneal chemotherapy for the initial management of primary epithelial ovarian cancer. Cochrane Database Syst Rev:CD005340.

Javid A, Ahmadian S, Saboury AA, Kalantar SM, Rezaei-Zarchi S, and Shahzad S. 2014. Biocompatible APTES-PEG modified magnetite nanoparticles: effective carriers of antineoplastic agents to ovarian cancer. Appl Biochem Biotechnol 173:36-54.

Kilkenny C, Browne WJ, Cuthill IC, Emerson M, and Altman DG. 2010. Improving bioscience research reporting: the ARRIVE guidelines for reporting animal research. PLoS Biol 8:e1000412.

Lammers T, Kiessling F, Hennink WE, and Storm G. 2012. Drug targeting to tumors: principles, pitfalls and (pre-) clinical progress. J Control Release 161:175-187.

Loira-Pastoriza C, Todoroff J, and Vanbever R. 2014. Delivery strategies for sustained drug release in the lungs. Adv Drug Deliv Rev 75:81-91.

Love RR, Leventhal H, Easterling DV, and Nerenz DR. 1989. Side effects and emotional distress during cancer chemotherapy. Cancer 63:604-612.

Lu Z, Tsai M, Lu D, Wang J, Wientjes MG, and Au JL. 2008. Tumor-penetrating microparticles for intraperitoneal therapy of ovarian cancer. J Pharmacol Exp Ther 327:673-682.

Mantia-Smaldone G, Ronner L, Blair A, Gamerman V, Morse C, Orsulic S, Rubin S, Gimotty P, and Adams S. 2014. The immunomodulatory effects of pegylated liposomal doxorubicin are amplified in BRCA1--deficient ovarian tumors and can be exploited to improve treatment response in a mouse model. Gynecol Oncol 133:584-590.

Massey RL, Kim HK, and Abdi S. 2014. Brief review: chemotherapy-induced painful peripheral neuropathy (CIPPN): current status and future directions. Can J Anaesth 61:754-762. 
689

690

691

692

693

694

695

696

697

698

699

700

701

702

703

704

705

706

707

708

709

710

711

712

713

714

715

716

717

718

719

720

721

722

723

724

725

726

727

728

729

730

731

732

733

734

735

736

737

738

739

Monsuez JJ, Charniot JC, Vignat N, and Artigou JY. 2010. Cardiac side-effects of cancer chemotherapy. Int J Cardiol 144:3-15.

Muggia FM, Hainsworth JD, Jeffers S, Miller P, Groshen S, Tan M, Roman L, Uziely B, Muderspach L, Garcia A, Burnett A, Greco FA, Morrow CP, Paradiso LJ, and Liang LJ. 1997. Phase II study of liposomal doxorubicin in refractory ovarian cancer: antitumor activity and toxicity modification by liposomal encapsulation. J Clin Oncol 15:987-993.

O'Brien ME, Wigler N, Inbar M, Rosso R, Grischke E, Santoro A, Catane R, Kieback DG, Tomczak P, Ackland SP, Orlandi F, Mellars L, Alland L, Tendler C, and Group CBCS. 2004. Reduced cardiotoxicity and comparable efficacy in a phase III trial of pegylated liposomal doxorubicin $\mathrm{HCl}$ (CAELYX/Doxil) versus conventional doxorubicin for first-line treatment of metastatic breast cancer. Ann Oncol 15:440-449.

Paraskar AS, Soni S, Chin KT, Chaudhuri P, Muto KW, Berkowitz J, Handlogten MW, Alves NJ, Bilgicer B, Dinulescu DM, Mashelkar RA, and Sengupta S. 2010. Harnessing structure-activity relationship to engineer a cisplatin nanoparticle for enhanced antitumor efficacy. Proc Natl Acad Sci U S A 107:12435-12440.

Perrault SD, Walkey C, Jennings T, Fischer HC, and Chan WC. 2009. Mediating tumor targeting efficiency of nanoparticles through design. Nano Lett 9:1909-1915.

$\mathrm{Pu}$ C, Chang S, Sun J, Zhu S, Liu H, Zhu Y, Wang Z, and Xu RX. 2014. Ultrasound-mediated destruction of LHRHa-targeted and paclitaxel-loaded lipid microbubbles for the treatment of intraperitoneal ovarian cancer xenografts. Mol Pharm 11:49-58.

Rapoport NY, Christensen DA, Fain HD, Barrows L, and Gao Z. 2004. Ultrasound-triggered drug targeting of tumors in vitro and in vivo. Ultrasonics 42:943-950.

Safra T, Groshen S, Jeffers S, Tsao-Wei DD, Zhou L, Muderspach L, Roman L, Morrow CP, Burnett A, and Muggia FM. 2001. Treatment of patients with ovarian carcinoma with pegylated liposomal doxorubicin: analysis of toxicities and predictors of outcome. Cancer 91:90-100.

Seetharamu N, Kim E, Hochster H, Martin F, and Muggia F. 2010. Phase II study of liposomal cisplatin (SPI-77) in platinum-sensitive recurrences of ovarian cancer. Anticancer Res 30:541-545.

Sengupta P, Basu S, Soni S, Pandey A, Roy B, Oh MS, Chin KT, Paraskar AS, Sarangi S, Connor Y, Sabbisetti VS, Kopparam J, Kulkarni A, Muto K, Amarasiriwardena C, Jayawardene I, Lupoli N, Dinulescu DM, Bonventre JV, Mashelkar RA, and Sengupta S. 2012. Cholesterol-tethered platinum II-based supramolecular nanoparticle increases antitumor efficacy and reduces nephrotoxicity. Proc Natl Acad Sci U S A 109:11294-11299.

Storm G, Nassander UK, Vingerhoeds MH, Steerenberg PA, and Crommelin DJA. 1994. Antibodytargeted liposomes to deliver doxorubicin to ovarian cancer cells. J Liposome Res 4:641-666.

Sugiyama T, Kumagai S, Nishida T, Ushijima K, Matsuo T, Yakushiji M, Hyon SH, and Ikada Y. 1998. Experimental and clinical evaluation of cisplatin-containing microspheres as intraperitoneal chemotherapy for ovarian cancer. Anticancer Res 18:2837-2842.

Tomasina J, Lheureux S, Gauduchon P, Rault S, and Malzert-Freon A. 2013. Nanocarriers for the targeted treatment of ovarian cancers. Biomaterials 34:1073-1101.

Tong L, Chen W, Wu J, and Li H. 2014. Folic acid-coupled nano-paclitaxel liposome reverses drug resistance in SKOV3/TAX ovarian cancer cells. Anticancer Drugs 25:244-254.

Truong J, Yan AT, Cramarossa G, and Chan KK. 2014. Chemotherapy-induced cardiotoxicity: detection, prevention, and management. Can J Cardiol 30:869-878.

Ueno N. 1988. Experimental studies on the chemotherapy of gynecological neoplasm by means of adriamycin entrapped in liposomes. Journal of the Aichi Medical University Association 16:6382.

Uziely B, Jeffers S, Isacson R, Kutsch K, Wei-Tsao D, Yehoshua Z, Libson E, Muggia FM, and Gabizon A. 1995. Liposomal doxorubicin: antitumor activity and unique toxicities during two complementary phase I studies. J Clin Oncol 13:1777-1785.

Vanderhyden BC, Shaw TJ, and Ethier JF. 2003. Animal models of ovarian cancer. Reprod Biol Endocrinol 1:67. 
Vergote I, Amant F, Kristensen G, Ehlen T, Reed NS, and Casado A. 2011. Primary surgery or neoadjuvant chemotherapy followed by interval debulking surgery in advanced ovarian cancer. Eur J Cancer 47 Suppl 3:S88-92.

Vergote I, Trope CG, Amant F, Kristensen GB, Ehlen T, Johnson N, Verheijen RH, van der Burg ME, Lacave AJ, Panici PB, Kenter GG, Casado A, Mendiola C, Coens C, Verleye L, Stuart GC, Pecorelli S, Reed NS, European Organization for R, Treatment of Cancer-Gynaecological Cancer G, and Group NCT. 2010. Neoadjuvant chemotherapy or primary surgery in stage IIIC or IV ovarian cancer. $N$ Engl J Med 363:943-953.

Vingerhoeds MH, Steerenberg PA, Hendriks JJ, Dekker LC, Van Hoesel QG, Crommelin DJ, and Storm G. 1996. Immunoliposome-mediated targeting of doxorubicin to human ovarian carcinoma in vitro and in vivo. Br J Cancer 74:1023-1029.

Werner ME, Karve S, Sukumar R, Cummings ND, Copp JA, Chen RC, Zhang T, and Wang AZ. 2011. Folate-targeted nanoparticle delivery of chemo- and radiotherapeutics for the treatment of ovarian cancer peritoneal metastasis. Biomaterials 32:8548-8554.

Winer I, Wang S, Lee YE, Fan W, Gong Y, Burgos-Ojeda D, Spahlinger G, Kopelman R, and Buckanovich RJ. 2010. F3-targeted cisplatin-hydrogel nanoparticles as an effective therapeutic that targets both murine and human ovarian tumor endothelial cells in vivo. Cancer Res 70:86748683.

Xu P, Van Kirk EA, Murdoch WJ, Zhan Y, Isaak DD, Radosz M, and Shen Y. 2006. Anticancer efficacies of cisplatin-releasing pH-responsive nanoparticles. Biomacromolecules 7:829-835.

Yang M, Yu T, Wood J, Wang YY, Tang BC, Zeng Q, Simons BW, Fu J, Chuang CM, Lai SK, Wu TC, Hung CF, and Hanes J. 2014. Intraperitoneal delivery of paclitaxel by poly(ether-anhydride) microspheres effectively suppresses tumor growth in a murine metastatic ovarian cancer model. Drug Deliv Transl Res 4:203-209.

Ye L, He J, Hu Z, Dong Q, Wang H, Fu F, and Tian J. 2013. Antitumor effect and toxicity of Lipusu in rat ovarian cancer xenografts. Food Chem Toxicol 52:200-206.

Yezhelyev MV, Gao X, Xing Y, Al-Hajj A, Nie S, and O'Regan RM. 2006. Emerging use of nanoparticles in diagnosis and treatment of breast cancer. Lancet Oncol 7:657-667.

Zeng Q, Wen H, Wen Q, Chen X, Wang Y, Xuan W, Liang J, and Wan S. 2013. Cucumber mosaic virus as drug delivery vehicle for doxorubicin. Biomaterials 34:4632-4642.

Zhang Y, Kenny HA, Swindell EP, Mitra AK, Hankins PL, Ahn RW, Gwin K, Mazar AP, O'Halloran TV, and Lengyel E. 2013. Urokinase plasminogen activator system-targeted delivery of nanobins as a novel ovarian cancer therapy. Mol Cancer Ther 12:2628-2639. 


\section{Figure 1 (on next page)}

\section{Figure 1}

Figure 1. Flow chart of study inclusion. PubMed and EMBASE via OvidSP were searched using developed search strings to identify studies that used chemotherapeutics in a DDS in ovarian cancer animal models. All studies were first screened by title and abstract according to predefined inclusion and exclusion criteria. Subsequently studies were more specifically assessed by full text. Screenings were performed by two reviewers (RR and WD). Full text studies excluded for "others" were: 1) no full text was available or only an abstract that did not include sufficient information $(n=12) ; 2)$ conference abstract of a previously assessed full-text study $(n=5) ; 3)$ the study included only a biodistribution experiment $(n=4)$. 
Removal of duplicates (788)

Combined search results (2735)

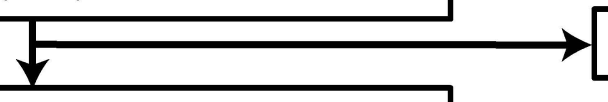

Records screened by title and abstract (1947)

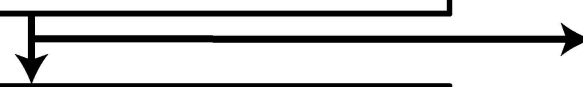

Excluded records by title and abstract (1682)

Records screened by full-text (265)

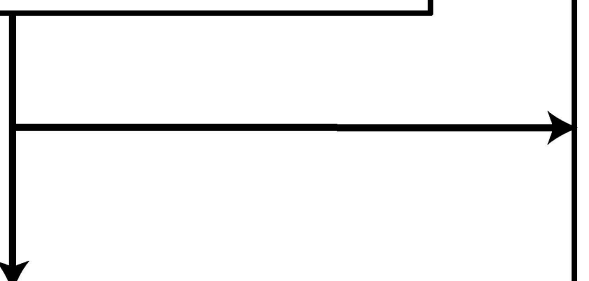

Excluded full-text studies (221):

1. No primary study (15)

2. Ovarian cancer cells used in other area

than peritoneal cavity or ovaries (128)

3. No drug delivery system (19)

4. Drug is not a chemotherapeutic (11)

$5 \quad$ Not ovarian cancer (14)

6. Drug not encapsulated (11)

7. Not an animal study (2)

Included in systematic review (44)

8. Others (21)

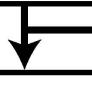




\section{Figure 2 (on next page)}

Figure 2

Figure 2. Risk of bias analysis. The risk of bias for all included studies was analyzed using several signaling questions. Depicted results are the answers for all studies per question. 


\section{PeerJ}

1. Is it mentioned that the experiment was randomised?

2. Is it mentioned that the experiment was blinded (level unknown)?

3. Is a power/sample size calculation shown?

4. Was the allocation sequence adequately generated and applied? 5. Were the groups similar at baseline or was adjusted for confounders in the analysis? 6. Was the allocation adequately concealed?

7. Were the animals randomly housed during the experiment? 8. Were the caregivers/ and or investigators during the course of the experiment blinded from knowledge of which intervention each animal received?

9. Were animals selected at random for the outcome assessment?

10. Was the outcome assessor blinded?

11. Were incomplete outcome data adequately addressed?

12. Was the study apparently free of other problems that could pose a high risk of bias?

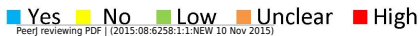




\section{Figure 3 (on next page)}

Figure 3

Figure 3. Effects of survival outcome measure of chemotherapeutics in a DDS compared to free chemotherapeutics (not in a DDS. A) The forest plot depicts hazard ratios with 95\% confidence interval $(\mathrm{Cl})$ and the weight of the study. A hazard ratio below 1 indicates a smaller chance for the animals to die over the course of the experiment due to treatment with chemotherapeutics in a DDS. A hazard ratio higher than 1 suggests that animals have a smaller chance of dying when treated with the free chemotherapeutic control condition. Statistical significance was reached when hazard ratios with their $95 \%$ confidence interval did not include the value of 1 . Numbers in brackets behind study names refer to details of the specific experiments; see supplementary material for details. B) Subgroup analysis for type of DDS, type of chemotherapeutic, targeted vs. non-targeted, IP vs. IV route of administration and inoculated cell type were performed. $\mathrm{n}$ is the number of experiments in the subgroups. 12 was used as a measure of heterogeneity. 


\section{Study name}

Chaudhury et al 2012 (1)

Yang et al 2014 (2)

Cirstoiu -11 사 ㅇt 2010 (3)

Cirstoiu-Hapca et al 2010 (4)

Konishi et al 2012 (5)

Pu et al 2014 (6)

Winer et al 2010* (7)

Cirstoiu-Hapca et al 2010 (8)

Shaikh et al 2013 (9)

Pastorino et al 2008 (10)

Pastorino et al 2008 (11)

Patankar et al 2013 (12)

$\mathrm{Pu}$ et al 2014 (13)

Winer et al 2010* (14)

Tong et al 2014 (15)

Cirstoiu-Hapca et al 2010 (16)

Tang et al 2012 (17)

Winer et al 2010* (18)

Lee et al 2013* (19)

Xiao et al 2009 (20)

Tong et al 2014 (21)

Li and Howell 2010* (22)

Rapoport et al 2004 (23)

Lu et al 2008 (24)

Tong et al 2014 (25)

Javid et al 2014 (26)

Javid et al 2014 (27)

Chaudhury et al 2012 (28)

Pu et al 2014 (29)

Pu et al 2014 (30)

\section{Hazard ratio $[95 \% \mathrm{CI}]$}

$0.00[0.00,6.219 \mathrm{E} 147]$

$0.00[0.00,3.860 \mathrm{E} 200]$

$0.00[0.00,3.244 \mathrm{E} 28]$

$0.00[0.00,3.355 \mathrm{E} 29]$

$0.02[0.00,0.56]$

$0.04[0.00,0.31]$

$0.04[0.01,0.24]$

$0.05[0.01,0.49]$

$0.07[0.01,0.71]$

$0.10[0.03,0.29]$

$0.15[0.05,0.42]$

$0.15[0.06,0.39]$

$0.18[0.03,0.98]$

$0.19[0.04,0.86]$

$0.21[0.07,0.63]$

$0.23[0.03,1.58]$

$0.31[0.12,0.76]$

$0.33[0.08,1.38]$

$0.41[0.13,1.26]$

$0.41[0.11,1.54]$

$0.41[0.15,1.12]$

$0.41[0.13,1.33]$

$0.43[0.09,1.98]$

$0.51[0.19,1.37]$

$0.87[0.67,1.14]$

$1.00[0.39,2.60]$

$1.00[0.38,2.62]$

$1.36[0.41,4.52]$

$3.95[0.98,15.93]$

$4.98[1.15,21.54]$

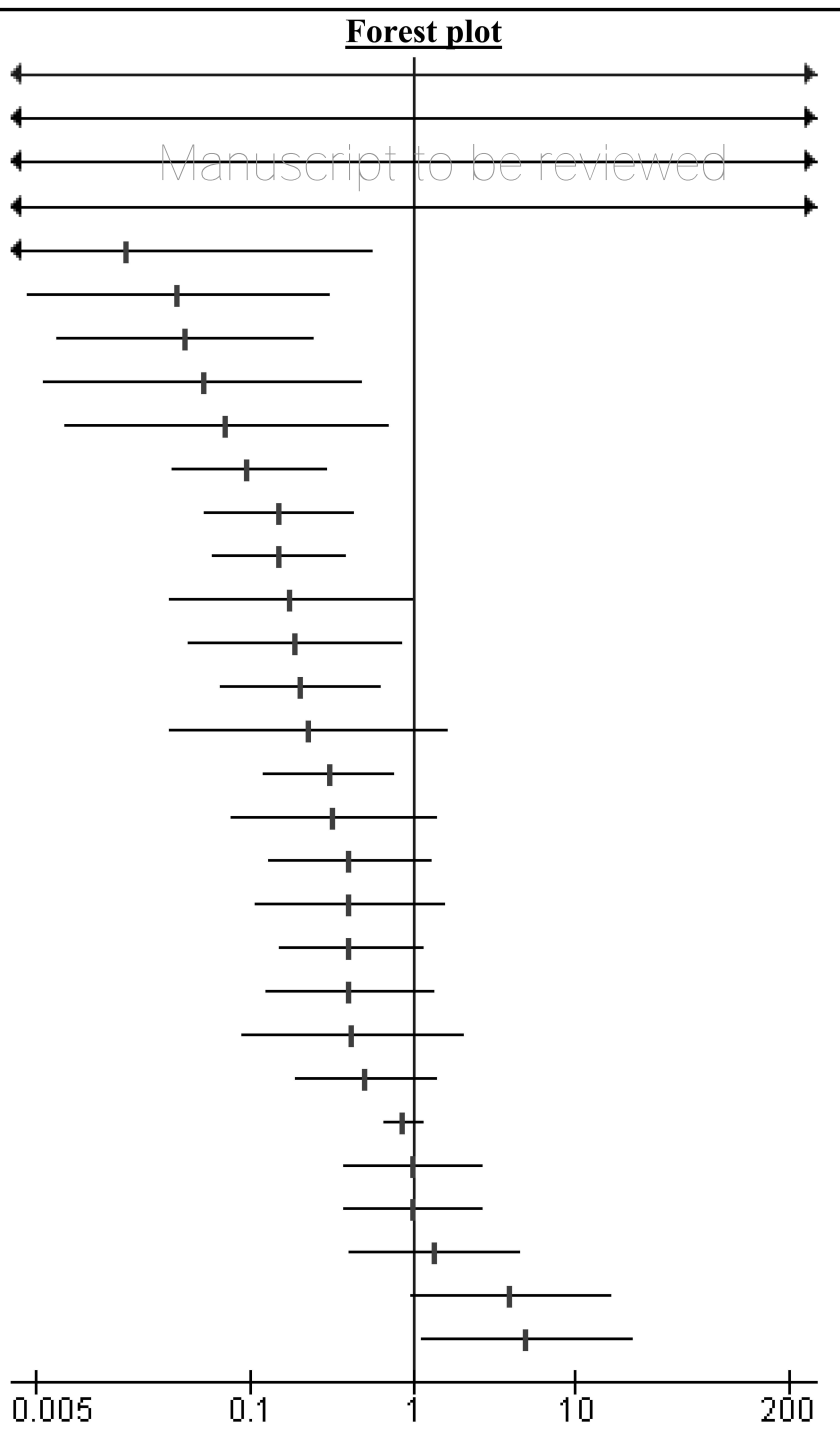

Favors chemotherapeutic in

B.

\begin{tabular}{|c|c|c|c|}
\hline \multicolumn{4}{|c|}{ Subgroups hazard ratio } \\
\hline Subgroup & Hazard ratio $[95 \% \mathrm{CI}]$ & $\underline{\mathbf{n}}$ & $\underline{I^{2}(\%)}$ \\
\hline Overall & $0.39[0.27,0.56]$ & 34 & 64 \\
\hline \multicolumn{4}{|l|}{ Type of DDS } \\
\hline Liposomes & $0.33[0.17,0.63]$ & 12 & 77 \\
\hline Micro/nano-particles & $0.38[0.17,0.83]$ & 15 & 68 \\
\hline \multicolumn{4}{|c|}{ Type of chemotherapeutic } \\
\hline Cisplatin & $0.36[0.18,0.70]$ & 7 & 51 \\
\hline Doxorubicin & $0.28[0.09,0.87]$ & 4 & 75 \\
\hline Paclitaxel & $0.56[0.38,0.84]$ & 20 & 53 \\
\hline \multicolumn{4}{|c|}{ Targeted vs. Non-targeted } \\
\hline Targeted & $0.24[0.10,0.57]$ & 12 & 79 \\
\hline Non-targeted & $0.48[0.33,0.69]$ & 22 & 46 \\
\hline \multicolumn{4}{|l|}{ IV vs. IP administration } \\
\hline IV & $0.21[0.08,0.50]$ & 9 & 84 \\
\hline IP & $0.55[0.36,0.84]$ & 19 & 52 \\
\hline Combination IP/IV & $0.24[0.11,0.51]$ & 6 & 0 \\
\hline \multicolumn{4}{|c|}{ Type of cell line Peerjreviewing PDF |(2015:08:6258:1:1:NEW 10 Nov 2015) } \\
\hline SKOV-3 & $0.41[0.23,0.73]$ & 9 & 49 \\
\hline OVCAR-3 & $0.46[0.27,0.79]$ & 9 & 55 \\
\hline A 2780 & $0.54[0.18,1.61]$ & 7 & 77 \\
\hline ID-8 & $0.15[0.05,0.48]$ & 3 & 39 \\
\hline IGROV-1 & $0.39[0.03,4.89]$ & 3 & 60 \\
\hline
\end{tabular}




\section{Figure 4 (on next page)}

Figure 4

Figure 4. Effects on tumor growth inhibition outcome measure of chemotherapeutics in a DDS compared to free chemotherapeutics (not in a DDS). A) The forest plot depicts SMDs with $95 \%$ confidence interval $(\mathrm{Cl})$ and the weight of the study. A statistically significant difference between interventional conditions (chemotherapeutic in DDS) and control conditions (chemotherapeutics not in a DDS) was reached when the SMD with its 95\% confidence interval was greater or smaller than zero. If below zero, the interventional condition is more efficient in reducing the tumor size, while if greater than zero, the control condition is more efficient in reducing the tumor size. Numbers in brackets behind study names refer to details of the specific experiments; see supplementary material for details. B) Subgroup analysis for type of DDS, type of chemotherapeutic, targeted vs. non-targeted and IP vs. IV route of administration were performed. $\mathrm{n}$ is the number of experiments in the subgroups. 12 was used as a measure of heterogeneity. 


\section{Study name}

Javid et al 2014 (1)

Javid et al 2014 (2)

Lu et al 2007 (3)

Ateh et al 2011 (4)

Ateh et al 2011 (5)

Patankar et al 2013-1*(6)

Zhang et al 2013 (7)

Yang et al 2014 (8)

Cirstoiu-Hapca et al 2010 (9)

Zhang et al 2013 (10)

Sengupta et al 2012 (11)

$\mathrm{Xu}$ et al 2006 (12)

Paraskar et al 2010 (13)

Ye et al 2013 (14)

Li and Howell 2010* (15)

Gilmore et al 2012 (16)

Xiao et al 2009 (17)

Jin et al 2007 (18)

Konishi et al 2012 (19)

$\mathrm{Xu}$ et al $2006(20)$

Jin et al 2007 (21)
SMD [95\% CI]

$-26.04[-34.14,-17.95]$

$-19.37[-25.42,-13.33]$

$-14.45[-18.43,-10.46]$

$-3.91[-6.95,-0.86]$

$-3.42[-6.18,-0.67]$

$-2.82[-4.60,-1.04]$

$-2.75[-4.57,-0.94]$

$-2.18[-3.92,-0.43]$

$-2.07[-4.63,0.49]$

$-1.82[-3.45,-0.19]$

$-1.67[-3.94,0.60]$

$-1.54[-3.80,0.72]$

$-1.43[-3.55,0.68]$

$-1.07[-2.32,0.18]$

$-1.04[-2.24,0.15]$

$-0.77[-1.76,0.22]$

$-0.71[-2.01,0.60]$

-0.07 [-1.46, 1.31]

Not estimable

$0.22[-1.49,1.93]$

$0.36[-1.05,1.77]$

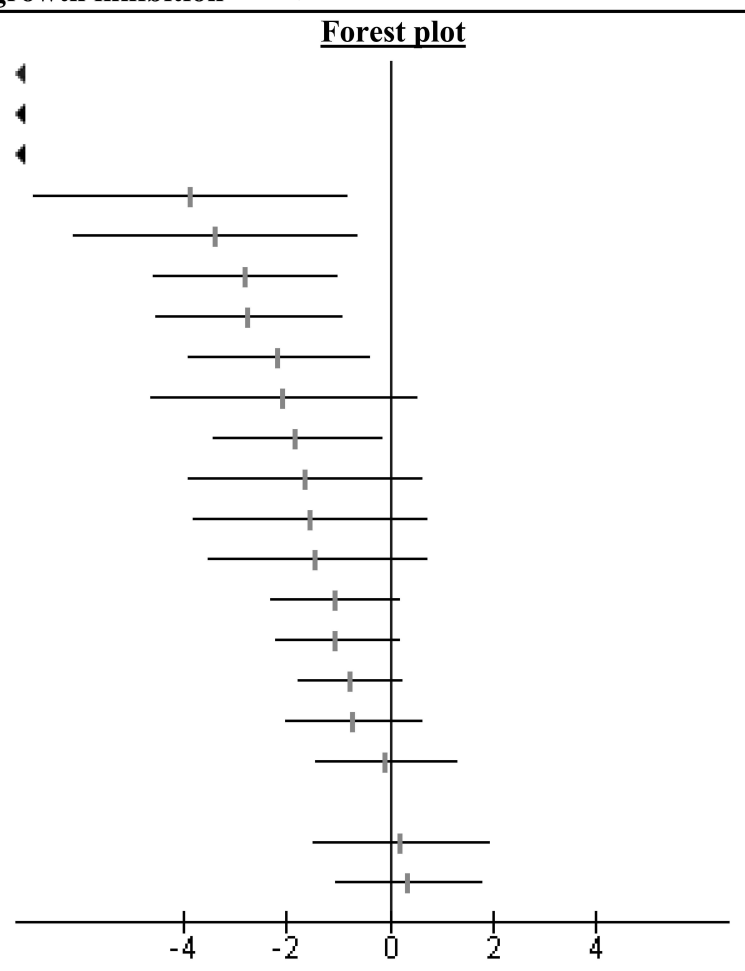

Favors chemotherapeutic in DDS
Favors free chemotherapeutic (not in a DDS)

B.

\begin{tabular}{|c|c|c|c|}
\hline \multicolumn{4}{|c|}{ Subgroups tumor growth inhibition } \\
\hline Subgroup & $\underline{\text { SMD }[95 \% \mathrm{CI}]}$ & $\underline{\mathbf{n}}$ & $\underline{I^{2}(\%)}$ \\
\hline Overall & $-2.70[-3.81,-1.59]$ & 21 & 86 \\
\hline \multicolumn{4}{|l|}{ Type of DDS } \\
\hline Micelles & $-0.57[-1.51,0.37]$ & 3 & 0 \\
\hline Liposomes & $-1.82[-3.52,-0.13]$ & 3 & 60 \\
\hline Micro/nano-particles & $-4.44[-6.24,-2.65]$ & 13 & 90 \\
\hline \multicolumn{4}{|c|}{ Type of chemotherapeutic } \\
\hline Cisplatin & $-0.54[-1.15,0.07]$ & 7 & 0 \\
\hline Paclitaxel & $-4.15[-6.20,-2.09]$ & 9 & 90 \\
\hline \multicolumn{4}{|c|}{ Targeted vs. Non-targeted } \\
\hline Targeted & $-2.05[-3.25,-0.86]$ & 4 & 33 \\
\hline Non-targeted & $-2.95[-4.31,-1.59]$ & 17 & 89 \\
\hline \multicolumn{4}{|l|}{ IV vs. IP administration } \\
\hline & -3 . & & $\begin{array}{l}\text { v } 2015) \\
89\end{array}$ \\
\hline
\end{tabular}


Figure $\mathbf{5}$ (on next page)

Figure 5

Figure 5. Funnel scatter plot of time-to-event studies. Hazard ratios with a $95 \%$ confidence interval were extracted and used to create a funnel scatter plot using Review Manager. Bullets represent individual experiments from included studies. The $x$-axis shows the hazard ratio and the $y$-axis represents the standard error of the log(hazard ratio). The funnel plot is missing studies in the bottom right area in which studies with a negative outcome are expected. Since there are no studies in this area, publication bias is suggested. 
